\title{
In Silico Approach To Design a B-cell Epitope Based Vaccine Target Against Yellow Fever Virus
}

\author{
Shamira Tabrejee ${ }^{1 *}$ and M. Mahboob Hossain ${ }^{2}$ \\ ${ }^{1}$ Biotechnology Program, Department of Mathematics and Natural Sciences, BRAC University, 66 Mohakhali, Dhaka-1212, Bangladesh, ${ }^{2}$ Microbiology \\ Program, Department of Mathematics and Natural Sciences, BRAC University, 66 Mohakhali, Dhaka-1212, Bangladesh,
}

\begin{abstract}
Yellow fever virusis a prototype member of the Flaviviridae family causing high fever and jaundice. Though YF 17D vaccine is administered to yellow fever patients, however it can produce adverse effects in immunocompromised, older people and young infants. The aim of this study is to design an epitope-based peptide vaccine by targeting envelope (E) protein of Yellow Fever Virus. Thirty sequences of $E$ protein of Yellow Fever Virus strains were retrieved from NCBI database. E protein was found to be mostly conserved among all the sequences with little variability and also was identified as a probable antigen. Different epitope prediction tools predicted 4 common epitopes, 3 of which were found to be antigenic. A peptide VKNPTDTGin E protein was predicted to have surface accessibility which overlaps with the VKNPTDTGHGT epitope.So, the whole VKNPTDTGHGT epitope was taken for further analysis.The VKNPTDTGHGT epitope showed $96.67 \%$ conservancy and also possesses flexibility, hydrophilicity and non-toxicity. Therefore, VKNPTDTGHGT can be regarded as a potential vaccine candidate against Yellow fever virus with further in vitro and in vivo validation.
\end{abstract}

Key words: Yellow fever virus, epitopes, peptide vaccine

\section{Introduction}

Yellow fever virus (YFV) belongs to the family Flaviviridae, genus Flavivirus. It is an arthropod-borne virus that can be transmitted by vectors such as ticks or mosquitoes, especially by Aedes aegypti mosquitos in humans. Yellow fever is an acute multisystem disease causing hepato-renal failure, profound jaundice and a bleeding diathesis. The mortality rate of yellow fever is even higher than dengue causing death in $20-50 \%$ of these cases ${ }^{1}$.There are 200,000 cases reported annually, which includes 30,000 deaths ${ }^{2}$. Yellow fever virus originated in Africaand 150 yellow fever outbreaks in 26 African countries were reported to World Health Organization (WHO) in between 1980 and 2012. Though yellow fever was not reported in Asia earlier, in 2016, 10 confirmed patients of yellow fever were detected in China who travelled from Angola, Africa. The abundance of primary mosquito vector Aedes aegypti, 20,000 Chinese workers along with many Indians and other Asians currently working in Angola, air travel through continents are conducive to the yellow fever introduction into Asia. This is also supported by the evidence that after a chikungunya virus outbreak in 2004 in Kenya which spreads via the same mosquito vector, India and Southeast Asia experienced an explosive epidemic of chikungunya transmitted to these regions via the Indian ocean basin ${ }^{1}$.

The YFV genome is single stranded, positive sense RNAencoding for a polyproteinwhich undergoes further processing in the endoplasmic reticulum and is broken down into three structural proteins (capsid, pM and E) and 7 non-structural proteins (NS1, NS2A, NS2B, NS3, NS4A, NS4B, NS5). The E protein is the most studied one due to its high antigenicity. It is crucial for various events such as viral attachment with host, penetration, fusion, host range and cell tropism ${ }^{3}$.

There is no clinically available antiviral drug against yellow fever virus, as a result prevention mainly depends on vaccination. Currently, a live attenuated vaccine known as $17 \mathrm{D}$ is inthemarketfor yellow fever virus. But it can give rise to yellow fever vaccine associatedviscerotropic diseases (YEL-AVD) and neurotropic diseases (YEL-AND). Young infants with immature blood brain barrier are more susceptible to neurotropic adverse events and 17D encephalitis ${ }^{4}$.

Epitopes are the antigenic determinants representing minimal immunogenic region of a protein antigen which elicit specific immune responses ${ }^{5}$. Epitope based vaccines could elicit effective immune responses against different pathogens like human immunodeficiency virus, multiple sclerosis, influenza virus, hepatitis $\mathrm{B}$ and $\mathrm{C}$ virus ${ }^{6-9}$. Development of epitope-based vaccines is easier compared to that of the traditional vaccine development. Moreover, epitope-basedvaccines are more specific and safe that are devoid of oncogenic and infectious hazard ${ }^{10}$.

The advent of computational tools has allowed the designing of conserved epitopes in antigenic proteins that may serve as 
vaccines against particular pathogens. This has been in turn facilitated by the availability of huge sequence data. The process will reduce the workload of wet lab studies by narrowing down the number of epitopes to be tested in vitro and in vivo, thus reducing time also. In this study, we predicted one B-cell peptide epitope in E protein of yellow fever virus using various bioinformatic tools that may serve as a universal vaccine against all yellow fever virus strains.

\section{Materials and Methods}

\section{Protein Sequence retrieval}

Thirtysequencesof E protein of Yellow Fever Virus were retrieved from NCBI database ${ }^{11}$ and downloaded in the FASTA format. The wild type isolates were found from various geographical locations and the size of $\mathrm{E}$ protein was found to be 493 amino acid residues.

\section{Variability analysis of E protein}

To find out the degree of conservation, all the sequences were aligned by EBI-Clustal Omega program ${ }^{12}$ and Multiple sequence alignment of the retrieved sequences was generated. Jalview ${ }^{13}$ was used to visualize the MSA and their absolute site variability was calculated by Shannon entropy analysis ${ }^{14}$ by using Protein Variability server $(\mathrm{PVS})^{15}$. Several variability metrices are utilized by PVS to calculate the sequence variability in a multiple sequence alignment.

\section{Prediction of antigenicity}

In order to develop an epitope-based vaccine, it is indispensable to check the antigenicity of $\mathrm{E}$ protein that would elicit an immune response. A reference $\mathrm{E}$ protein sequence with an accession number AAA92692.1 was tested for its antigenicity. The antigenicity was checked by using Vaxijen v2.0 server ${ }^{16}$ and Kolaskar\&Tongaonkar method ${ }^{17}$.Vaxijen is the first server for alignment-independent prediction of protective antigens. It was developed to allow antigen classification solely based on the physicochemical properties of proteins without recourse to sequence alignment.

\section{Linear B cell prediction}

$B$ cell epitope predictions can be done with various servers each having their own strengths and weaknesses. Therefore, two most popular epitope prediction tools were used such as BepiPred 1.0 18 and BCPREDS ${ }^{19}$ to minimize false positive predictions. BepiPred 1.0server uses the Hidden Markov Model to predict the epitope along with the propensity scale, with a threshold for the prediction set at 0.35 for viruses. BCPREDS was used to predict nonoverlapping and fixed length epitopes of 12 amino acids with a specificity of $75 \%$. The predicted epitopes from both servers were compared manually and the common epitopes were taken for further analysis.

\section{Conservancy analysis of B cell epitope}

Conservancy of the epitopes was analyzed by the IEDB conservancy analysis too ${ }^{20}$. For calculating the conservancy score the sequence identity threshold was kept at $80 \%$.

\section{Surface accessible regions prediction}

In order to determine the surface accessible regions of E protein, Emini surface accessibility prediction tool of IEDB was used ${ }^{21,22}$. The accessible regions were later compared to the conserved $\mathrm{B}$ cell epitopes. Common peptides from both of the servers were selected for further investigation.

\section{Prediction of flexibility and hydrophilicity of B cell epitopes}

The conserved epitopes were subjected to Karplus and Schulz (KS) flexibility prediction ${ }^{23}$, and Parker hydrophilicity prediction tools ${ }^{24}$ for flexibility and hydrophilicity analyses respectively. For predicting protein flexibility, the KS method uses normalized $\mathrm{B}$-values of $\mathrm{C} \pm$-atoms in protein structures. Due to its robustness, it has been widely used for the analysis of protein flexibility ${ }^{25,26,27}$.

\section{Prediction of toxicity of the $B$ - cell epitopes}

Anidealepitope should have no or less toxicity ${ }^{28}$. Hence, to determine the toxicity of the selected B-cell epitopes, Toxin Pred web server was used ${ }^{29}$.

Prediction of the tertiary structure of E protein using in silico approach

The tertiary structure of the E protein was predicted using SWISS MODEL server ${ }^{30}$. Template selection, target template alignment, model building and evaluation are the steps of this homologymodeling method ${ }^{31}$. The model was then evaluated by ROCHECK software which constructed Ramachandran plot for the $\mathrm{E} \mathrm{protein}^{32}$. Ramachandran plot analyzes residue by residue geometry and overall structure geometry, thus assesses the stereochemical quality of the 3-D structure. For further quality evaluation of the 3-D structure,ProSA web tool was used which provides a $Z$-score measuring the quality of the $3-\mathrm{D}$ model ${ }^{33}$.

\section{Validation of the workflow}

Since the work flow used here includes various computational tools developed by different platforms, so the validation of the workûowwasrequired. A conserved B-cell epitope, SVQYHPL, was identified from the envelope protein of the reticuloendotheliosis virus through wet lab screening ${ }^{34}$. The sequence of that envelope protein was retrieved from the NCBI data base and fedinto the work flow to check whether the work flow can identify and qualify that as a B-cell epitope.

\section{Results}

E protein is well conserved in all pathogenic Yellow Fever virus strains

In order to determine the conserved region, multiple sequence alignment (MSA) using Clustal Omega ${ }^{12}$ and Protein variability analysis ${ }^{15}$ were performed. MSA (Figure 1) and protein variability index (Figure 2) of these proteins identified that amino acids positioned from 108 to 445 are conserved among all the sequences. 

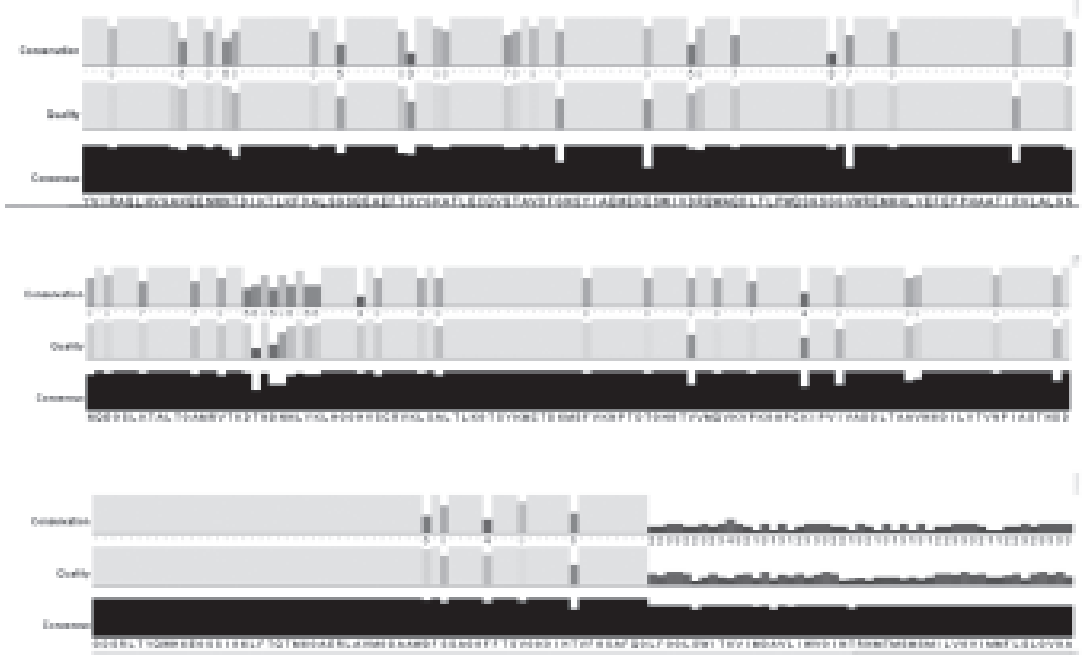

Figure 1. Multiple sequence alignment of the 30 sequences of E protein of yellow fever viruses visualized by Jalview 2.8. This tool provides a bar chart summarizing the conservation using 11-base scale for conservancy and BLOSUM 62 for quality along with the alignment. Yellow color bar and star sign indicate the full conservation. Black bars show the consensus sequence and yellow color indicates good quality.

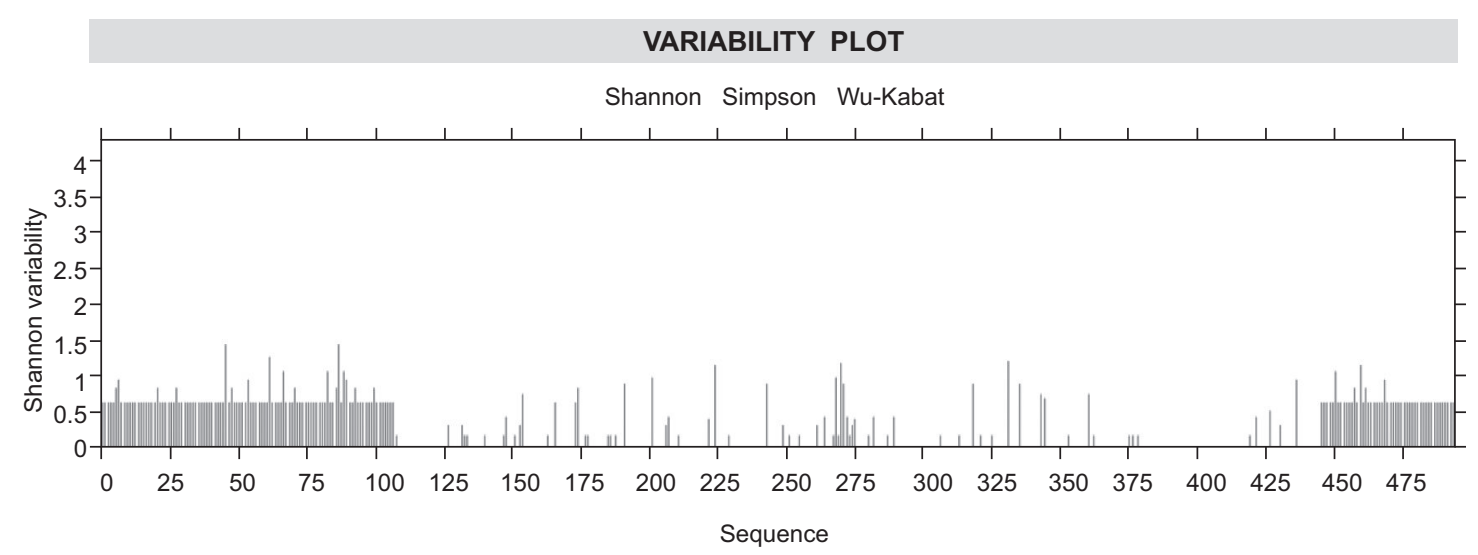

Figure 2. Protein variability index of E protein by using PVS server. The threshold of conservancy was kept at 1.0 at this analysis. $X$ axis indicates the position of amino acid sequence and $Y$ axis indicates the Shannon entropy.

\section{E protein is antigenic}

In order to become a vaccine candidate, a protein sequence must be antigenic to mount an immune response. The antigenicity of E protein sequence with accession number AAA92692.1 was found to be 0.5794 by using Vaxijen v2.0 server ${ }^{16}$. A threshold of 0.4 was taken as a default for viruses. Kolaskar\&Tongaonkarantigenicity prediction tool ${ }^{17}$ predicted 341 amino acids to be above the threshold of 1.0. The maximum and minimum scores were 1.238 (at position 62) and 0.830 (at position 470) respectively with an average of 1.028 . The window size was kept at 7 with a central position of 4 (Figure 3).

Four epitopes are found to becommon by all the prediction tools employed

Two widely used epitope prediction tools Bepipred and BCPREDS ${ }^{18,19}$ were used for epitope prediction. Bepi Predpredicted ten epitopes with a size range taken to be equal or above six amino acids, whereas BCPREDSpredicted 14 epitopes, each having a length of twelve amino acids. Four common epitopes were found between them and their antigenicity was checked by Vaxijen v2.0 server with a threshold 0.4 (Table 1). The positions of these epitopes in the E protein are: 146-154; 249-255; 263-271; 307-317.

One B-cell epitope is highly conserved among the E protein sequences of Yellow Fever Virus

Conservancy is a percentile measure of our epitope's availability in all the retrieved sequences. Among four common predicted epitopes, three were antigenic and one was not predicted to have antigenicity by Vaxijen v2.0 server. So only those three antigenic epitopes, GANQENWNA, TKDTNGS and VKNPTDTGHGT were analyzed for conservancy by IEDB conservancy analysis tool $^{20}$. Among those three, only one epitope was found to be conservedwith an identity of $96.67 \%$. It is $100 \%$ conserved in 29 out of 30 sequences.(Table 2) 
Tabrejee et. al

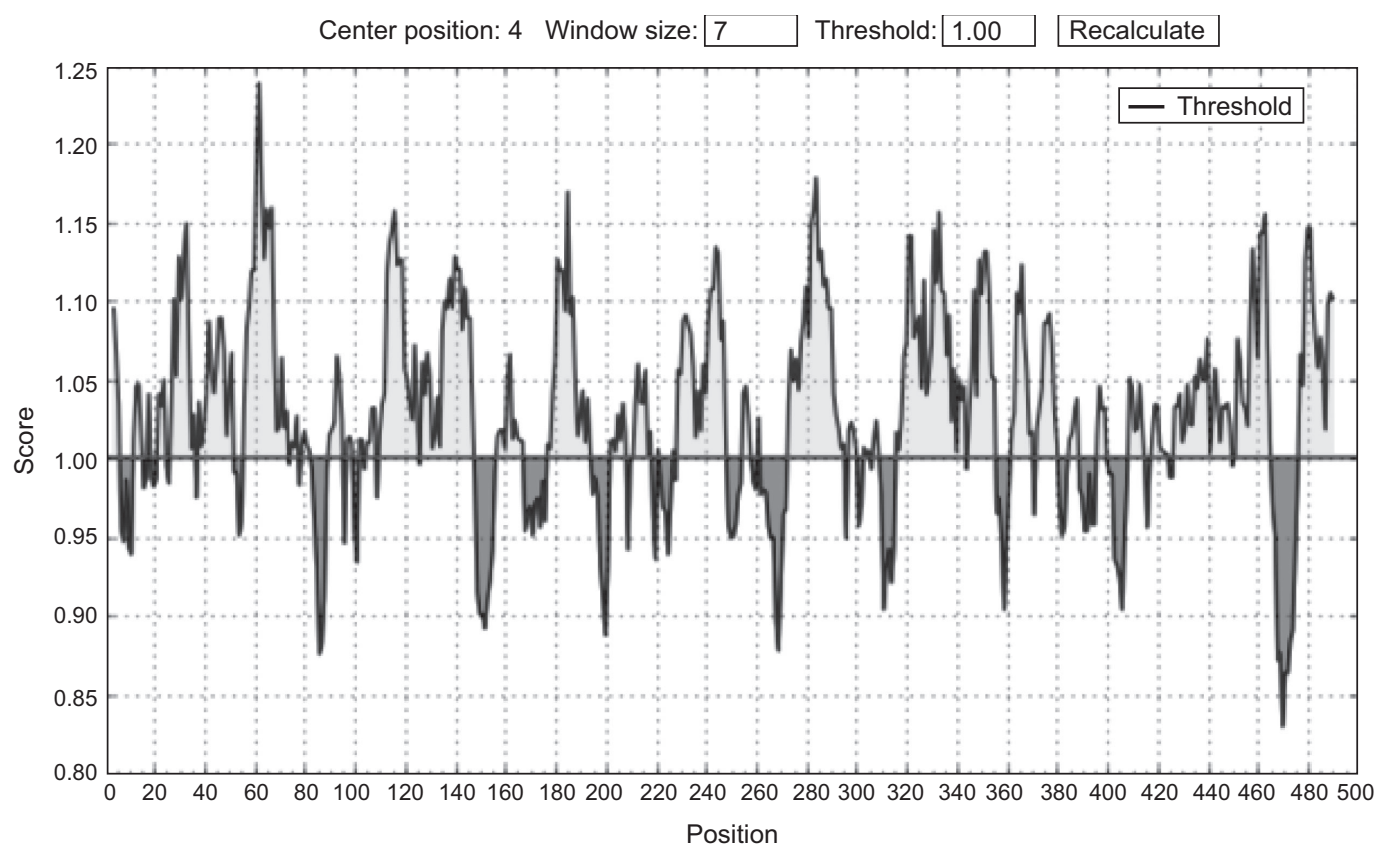

Figure 3. The E protein is found to be antigenic. The threshold was kept at 1.0 indicated by the red line. The yellow regions indicate antigenic residues in nature and green regions indicated non-antigenic regions.

Table 1. Four common epitopes between BepiPred and BCPREDS along with their length, position and antigenicity

\begin{tabular}{llcccc}
\hline No. & Epitope & Length & Position in the E protein & Antigenicity value & Presence of antigenicity \\
\hline 1 & GANQENWNA & 9 & $146-154$ & 0.7463 & Yes \\
2 & NQEGSLK & 7 & $249-255$ & -0.1216 & No \\
3 & TKDTNGS & 9 & $263-271$ & 0.7006 & Yes \\
4 & VKNPTDTGHGT & 11 & $307-317$ & 1.0508 & Yes \\
\hline
\end{tabular}

Table 2. Conservancy of common antigenic epitopes

\begin{tabular}{|c|c|c|c|c|}
\hline$\overline{\text { No. }}$ & Epitope & $\begin{array}{c}\text { Percent of protein sequence matches } \\
\text { at identity }<=100 \%\end{array}$ & Minimum identity & Maximum identity \\
\hline 1 & VKNPTDTGHGT & $96.67 \%(29 / 30)$ & $90.91 \%$ & $100 \%$ \\
\hline 2 & GANQENWNA & $3.33 \%(1 / 30)$ & $55.55 \%$ & $100 \%$ \\
\hline 3 & TKDTNGS & $20.00 \%(6 / 30)$ & $57.14 \%$ & $100 \%$ \\
\hline
\end{tabular}

\section{Eleven surface accessible peptides are predicted}

Emini surface accessibility prediction tool ${ }^{22}$ was used to determine the surface accessible peptides within the E protein above a threshold cut off 1.0 (Figure 4). Eleven such peptides above thethreshold were detected and compared with the predicted epitopes (Table 3 ). The maximum and minimum scores were 5.814 (at position 95) and 0.065 (at position 480) respectively. The window size was kept at 6 with a central position of 3.Among these eleven peptides VKNPTDTG overlaps with the BepiPred and BCPREDS predicted consensus and conserved epitope VKNPTDTGHGT. Asa result, the whole VKNPTDTGHGT epitope was taken for further analysis.

\section{VKNPTDTGHGTis flexible and hydrophilic}

Flexibility and hydrophilicity are the properties of being an ideal epitope. VKNPTDTGHGT was found to be highly flexible by Karplus and Schulz prediction analysis ${ }^{23}$ (Fig 5). It was also found to be hydrophilic in nature by IEDB Parker hydrophilicity analysis $^{24}$ (Fig 6). The maximum and minimum scores were 1.067 and 1.045 for flexibility prediction and 5.843 and 4.5 for hydrophilicity prediction respectively. The window size was kept at 7 with a central position of 4 for both of the analyses. Hence, VKNPTDTGHGT epitopefulfilsall the main properties of being an ideal epitope candidate. 


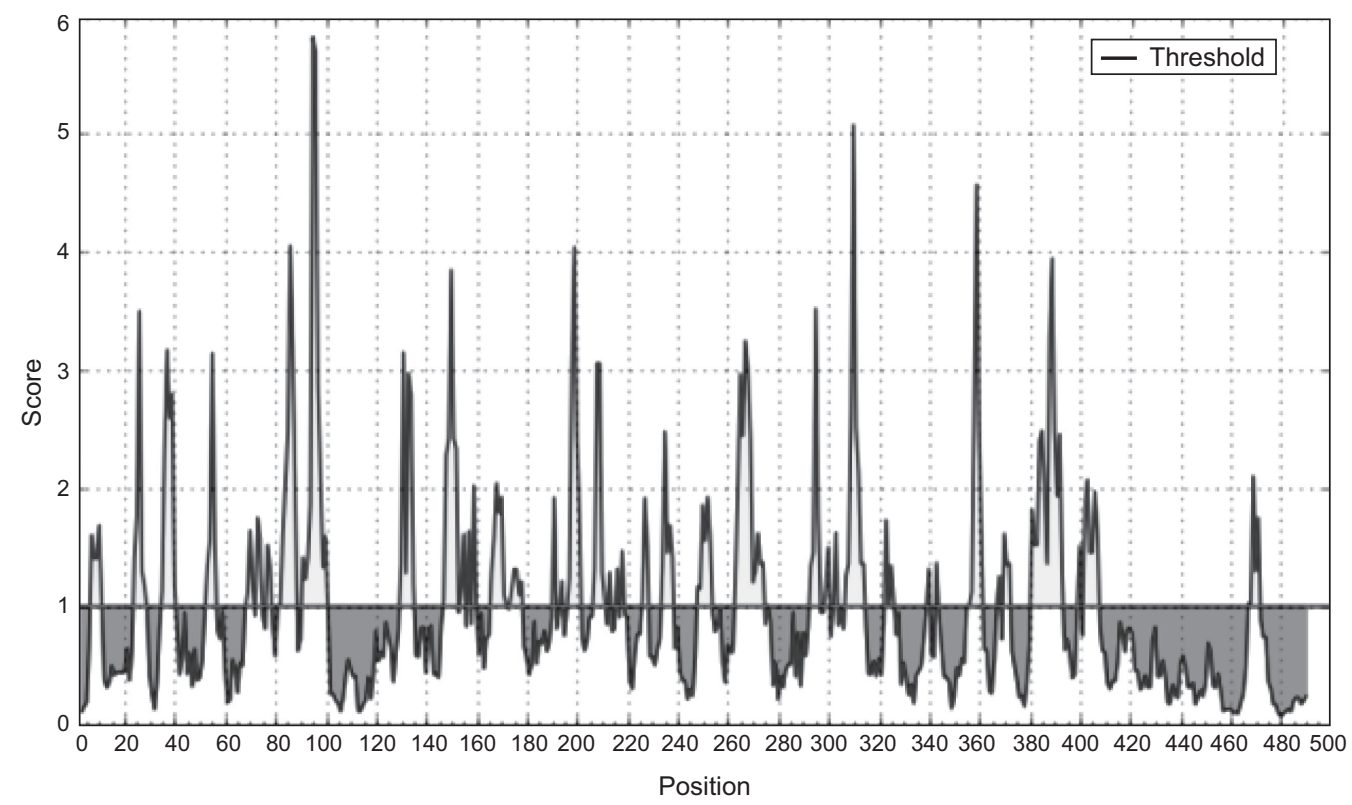

Figure 4. Surface accessibility of the E protein. The threshold was kept at 1.0 indicated by the red line. The surface accessible residues are indicated by the yellow regions above the threshold cutoff.

Table 3. Predicted surface accessible antigenic sites by using Emini surface accessibility prediction analysis

\begin{tabular}{llcc}
\hline No. & Peptide & Length & Position in the E protein \\
\hline 1 & APDKPS & 6 & $35-40$ \\
2 & HLAEENEG & 8 & $81-88$ \\
3 & ACKRTYSDRG & 10 & $91-100$ \\
4 & VDQTKI & 6 & $130-135$ \\
5 & ANQENW & 6 & $147-152$ \\
6 & GSQEAE & 6 & $166-171$ \\
7 & GNQEGS & 6 & $248-253$ \\
8 & RVTKDTNGSNLY & 12 & $263-274$ \\
9 & VKNPTDTG & 8 & $307-314$ \\
10 & GDSRLTYQWHKEG & 13 & $381-393$ \\
11 & QTMKGA & 6 & $402-407$ \\
\hline
\end{tabular}

\section{VKNPTDTGHGT is non-toxic}

The toxicity of the VKNPTDTGHGT epitope was analyzed by the ToxinPred server ${ }^{29}$ and was found to be non-toxictocell provingits potential as a candidate vaccine.

\section{Tertiary structure of E protein was predicted and validated}

As the full experimental tertiary structure of the E protein of Yellow fever virus is not available, in this study a tertiary structure was modelled by homology modelling ${ }^{30}$. Two models were generated by SWISS MODEL server. Among those the best model was selected based on GMQE and QMEAN score, for which the template was 4 fg0.1.A (Fig 7). When the model was validated by Ramachandran plot generated by PROCHECK software, it was found that $87.2 \%$ residues were in the favourable region and the average G- factor was - 0.1 (Fig 8A). Z-score by ProSA was calculated to check whether the input structure is within the range of scores typically found fora native protein of similar size ${ }^{33}$. Predicted Z-score was- 8.66 which indicatedthe overall good quality of the model (Fig 8B). Along with the analysis of surface accessibility, flexibility and hydrophilicity, thetertiary structure also predicted that the VKNPTDTGHGT epitope is present on the surface of the E protein of Yellow fever virus (marked as blue colour in the 3D model).

\section{Designed workflow concords with the experimental results}

The workûow, designed to predict epitopes, used here was validated by positive controls. Xue et al., in 2012, mapped a linear B-cell epitope, SVQYHPL, located in the envelope protein of Reticuloendotheliosis virus ${ }^{34}$. When the sequence of the envelope protein was fed into the workûow for the B-cell epitope, the same B-cell epitope, SVQYHPL, was successfully identified. 


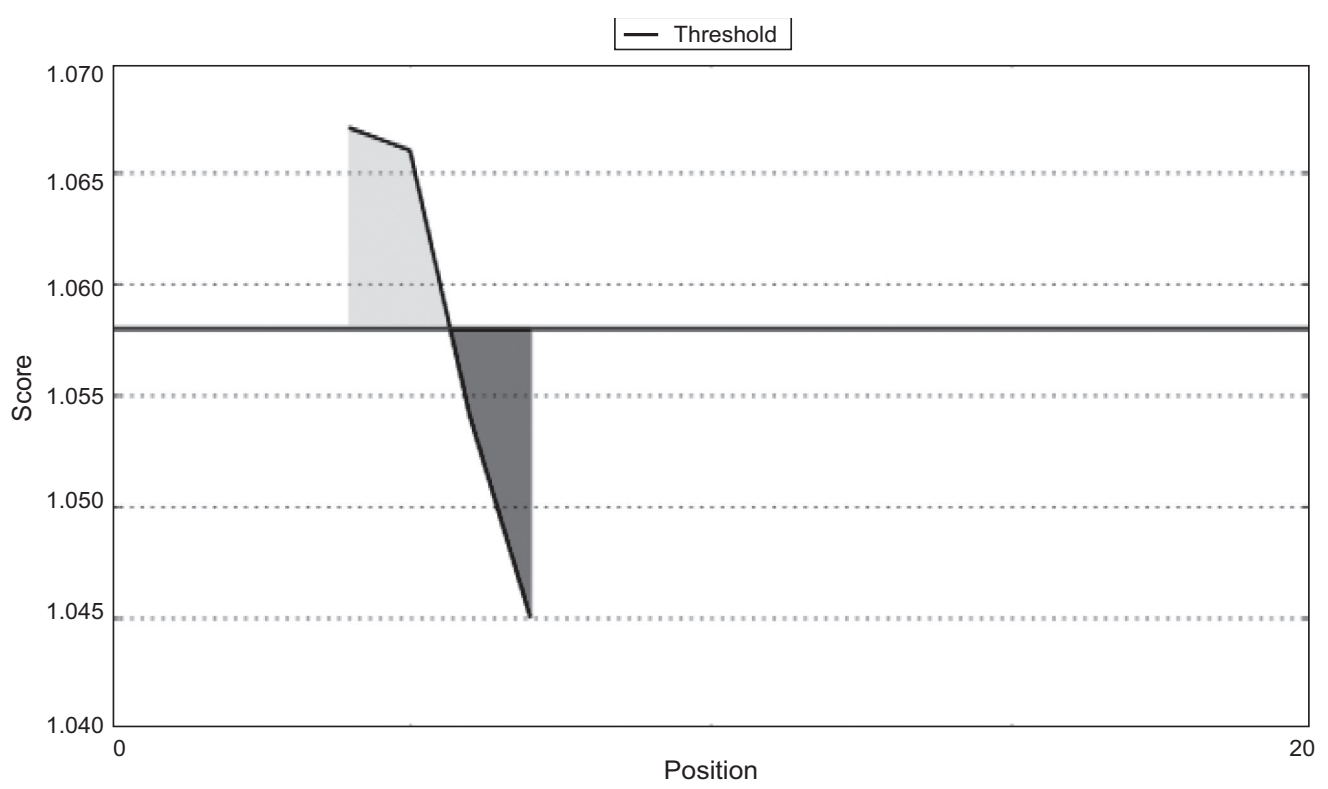

Figure 5. Flexibility of VKNPTDTGHGT epitope. Most of the residues of the desired VKNPTDTGHGT epitope were found to be flexible in nature (in the yellow colored region). The residues which are below the cut off 1.058 (red line) are in the green region.

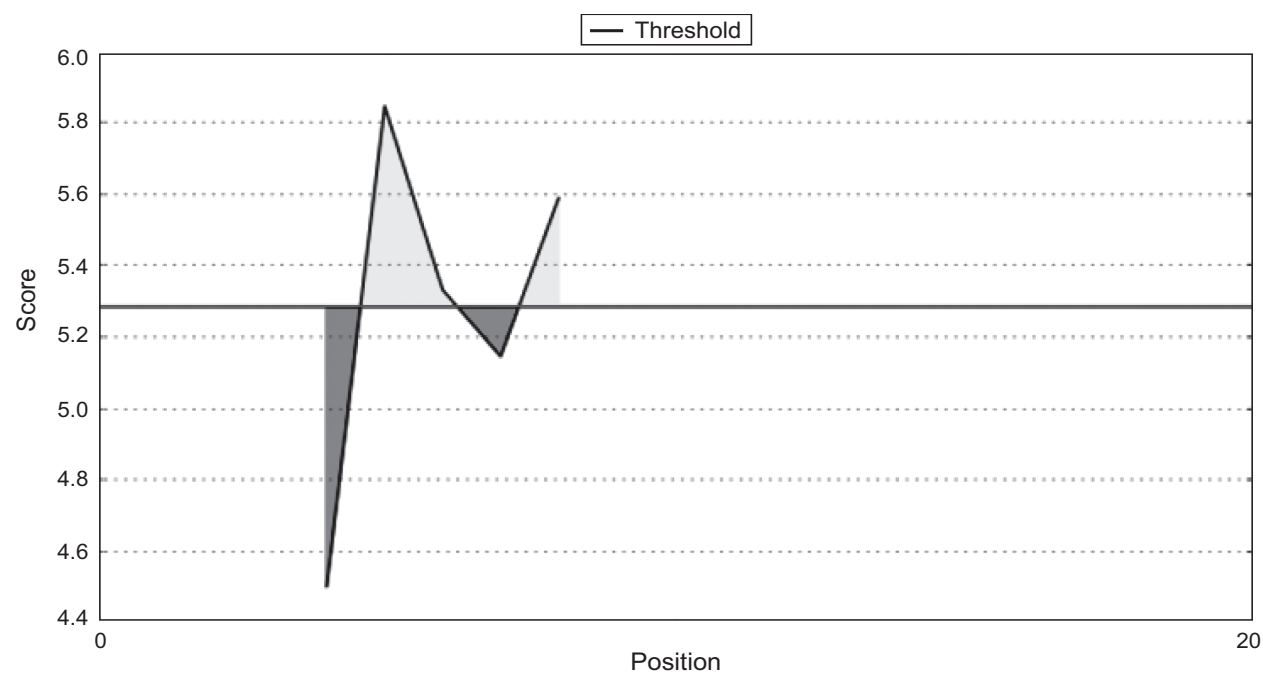

Figure 6. Hydrophilicity of VKNPTDTGHGT epitope. Most of the residues of the desired VKNPTDTGHGT epitope were found to be hydrophilic in nature (in the yellow colored region). The residues which are below the cut off 5.280 (red line) are in the green region.

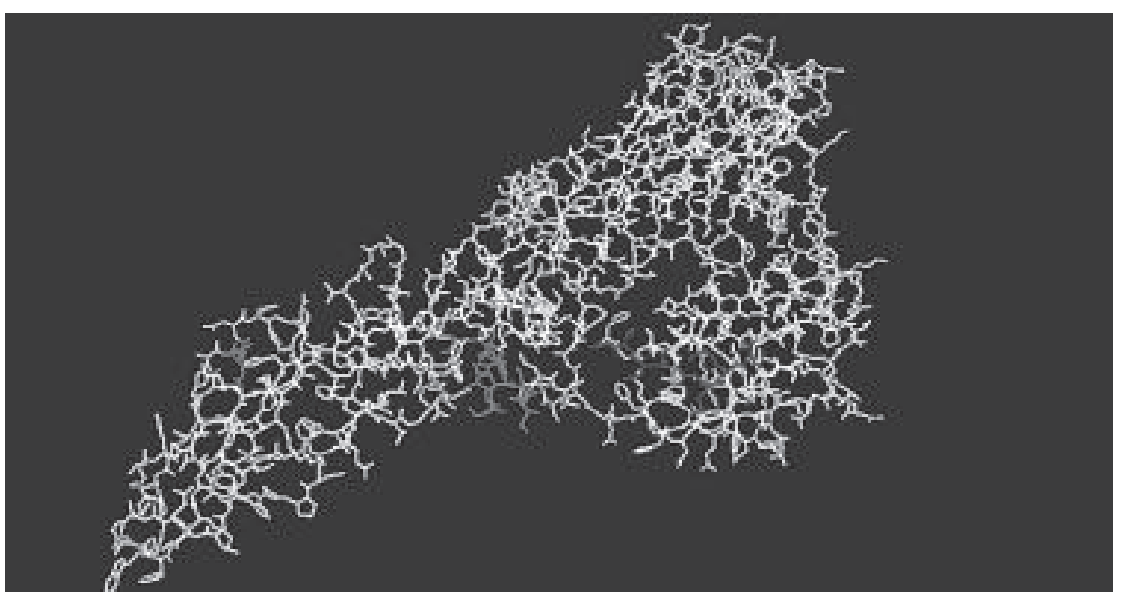

Figure 7. 3D structure of the E protein. Predicted conserved VKNPTDTGHGT epitope was mapped on the E protein using SWISSPdbViewer ${ }^{47}$. The blue colouredpeptide region of the $3 D$ model represents the conserved VKNPTDTGHGT epitope 

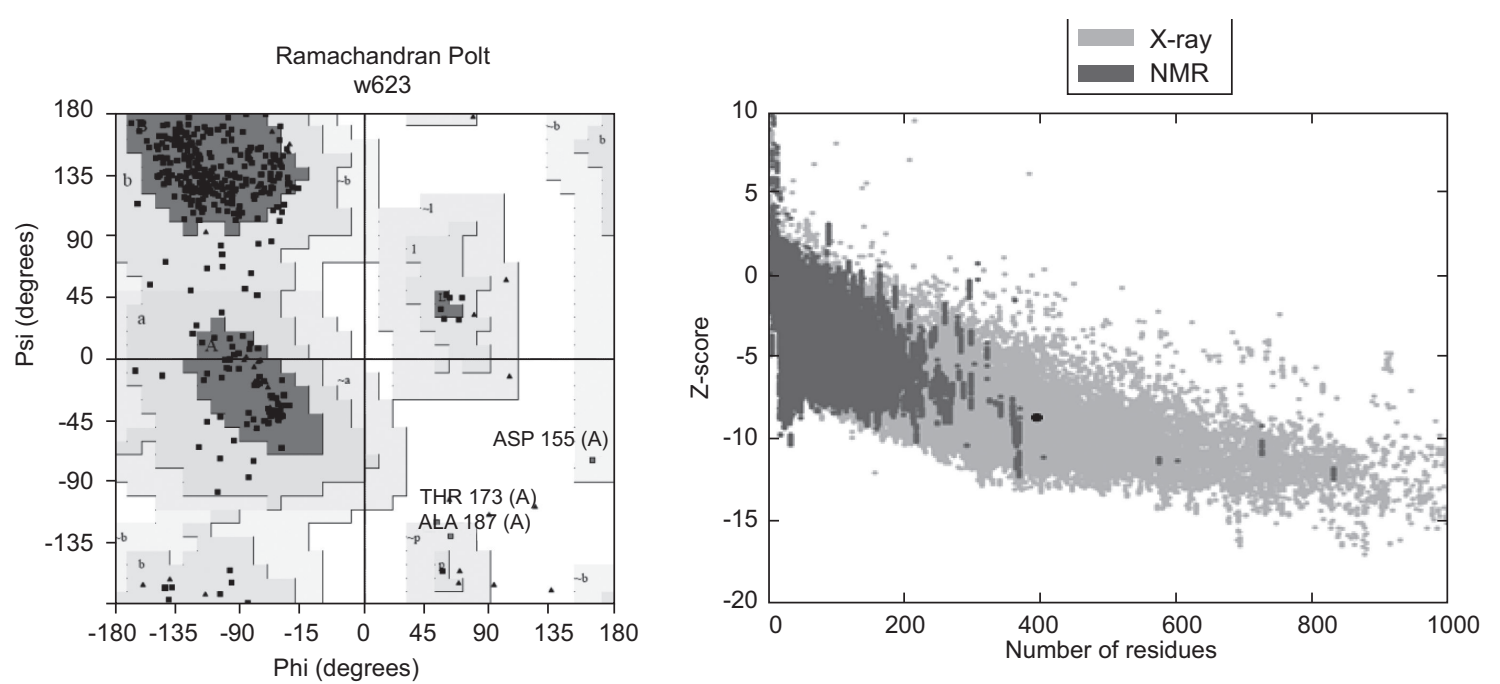

\section{Plot statistics}

\begin{abstract}
Residues in most favoured regions [A,B,L] Residues in additional allowed regions [a,b.,p] Residues in generously allowed regions $\left[-a_{,}-b,-1,-p\right]$ Residues in disallowed regions

Number of non-glycine and non-proline residues

Number of end-residues (excl. Gly and Pro)

Number of glycine residues (shown as triangles) Number of proline residues
\end{abstract}

Total number of residues

\begin{tabular}{rr}
300 & $87.2 \%$ \\
41 & $11.9 \%$ \\
2 & $0.6 \%$ \\
1 & $0.3 \%$ \\
\hline 344 & $100.0 \%$ \\
2 & \\
35 & \\
14 & \\
\hline 395 &
\end{tabular}

Figure 8. Ramachandran plot along with statistics showing residues in the most favorable, additionally allowed, generously allowed and disallowed regions (A). Z-score for quality of the $3 D$ structure E protein by ProSA (B).

\section{Discussion}

Yellow fever has been a major threat to human health since $18^{\text {th }}$ Century with repeated epidemics and dispersion from the endemic areas to coastal towns and cities ${ }^{4}$. In 2016 and 2017, 576 confirmed cases of yellow fever including 184 deaths were reported in Brazil. In 2017 and 2018, the number of confirmed cases increased to 723 with 237 deaths in the same region $^{35}$.Thougheffective17D and 17DD vaccines prevail against yellow fever viruses, those showed fatal side effects in some cases $^{36-38}$. The lethality of the adverse events of $17 \mathrm{D}$ vaccine associated viscerotropic disease is higher than that of the wildtype yellow fever ${ }^{4}$. Moreover, the vaccine is not recommended for pregnant women, infants and immunocompromised people ${ }^{39}$. In the U.S an inactivated 17D vaccine was produced and tested clinically but failed to go for commercialization ${ }^{4}$. Two non-viral DNA based antigens were also evaluated as vaccine candidates and revealed potency but further development studies are required ${ }^{40}$.Considering these factors, the idea of designing an alternative vaccine for yellow fever virus to replace or complement the existing live attenuated one can be pursued.

With theever-progressing knowledge of antigen recognition by antibodies at the molecular level, a new window for vaccine designing and development has opened, which overcomes the drawbacks of the traditional vaccine development processes. Though several T-cell epitope regions have been discovered in $\mathrm{E}$ protein ${ }^{40}$, however no B-cell epitope has been reported so far.In this study, we aimed to design potential B-cell epitopes which can be used universally against all the strains of yellow fever viruses. The main benefit of immunization with an epitope-based vaccine is its ability to immunize with a minimal structure that is able to stimulate an effective specific immune response, while avoiding potential undesirable effects ${ }^{41}$. In this study we have chosenE protein over others because it contributed in immunological antiviral response by eliciting neutralizing antibodies $^{42}$.

The first challenge to design an epitope-based vaccine is to confirm the conservancy of the candidate protein and find out the low variability regions in the sequences. In addition to that, the envelope protein has a tendency to undergo frequent mutations to evade host defense. Hence, we determined the conservancy of E protein by MSA and protein variability server that identified almost $70 \%$ residues of E protein to be conserved. We also found $70 \%$ of the amino acids in E protein are antigenic.For predicting B-cell epitopes, we used two B-cell epitope prediction tools to 
avoid false positive results. This is because, though several Bcell epitope prediction methods are available, their efficacy to identify epitopes may far from ideal ${ }^{43}$. Keeping this in mind, we only considered four common epitopes predicted by both of the servers, but only three of them proved to be antigenic in further evaluation. When conservancy was checked for the individual epitopes, it was revealed that only VKNPTDTGHGT epitope among the three is well conserved among the strains of yellow fever virus. To allow an antibody to bind with an epitope and induce immune response, the epitope should be accessible for the antibody ${ }^{44}$. We identified 11 surface accessible peptides in E protein, among which the surface accessible VKNPTDTG peptide and the previously predicted epitope VKNPTDTGHGT were found to be located in the same region of the E protein.So, the whole VKNPTDTGHGT peptide was taken for consideration. The three-dimensional validated structure of $\mathrm{E}$ protein also showed that the epitope is present on the surface of the protein, hence it should be more accessible. This epitope also revealed to have good flexibility and hydrophilicity which strengthens its potency as a good vaccine candidate as flexibility and hydrophilicity are two characteristic features of an ideal epitope ${ }^{45}$. An added value of the VKNPTDTGHGT epitope as a vaccine is; it is predicted to be a non-toxic peptide, thus having no possibility to generate adverse reactions inside the body.

All the data retrieved from our analysis hold promises to use the VKNPTDTGHGT epitope as a universal vaccine against yellow fever virus. Adjuvants might be added to increase its immunogenicity and stability ${ }^{46}$. As this vaccine has been designed by in silico analyses, the actual immunogenicity, efficacy, stability and delivery strategies in humans should be checked by in vitro and in vivo studies.

\section{References}

1. Wasserman S, Tambyah PA, Lim PL. 2016. Yellow fever cases in Asia: primed for an epidemic. 2016. Int J Infect Dis. 48: 298-103.

2. Pramil T, Rajiv A, Gaurav G. 2016. Safety of yellow fever vaccine in Indian travellers: A prospective observational study. Indian J Med Res. 144(5): 778-780.

3. Chávez JH, Silva JR, Amarilla AA, Figueiredo LTM. Domain III peptides from flavivirus envelope protein are useful antigens for serologic diagnosis and targets for immunization. 2010. Biologicals. 38: 613-618.

4. Monath TP, Vasconcelos PFC. 2015. Yellow Fever. J Clin Virol. 64:160173.

5. Purcell AW, McCluskey J, Rossjohn J. 2007. More than one reason to rethink the use of peptides in vaccine design. Nat Rev Drug Discov. 6(5):404-14.

6. Wilson CC, McKinney D, Anders M, MaWhinney S, Forster J, Crimi C et al. 2003. Development of a DNA vaccine designed to induce cytotoxic T lymphocyte responses to multiple conserved epitopes in HIV-1. $J$ Immunol. 171(10):5611, 2003.

7. Bourdette DN, Edmonds E, Smith C, Bowen JD, Guttmann CR, Nagy ZP et al. 2005. A highly immunogenic trivalent $T$ cell receptor peptide vaccine for multiple sclerosis. MultScler. 11(5): 552-561.

8. Staneková Z, Vare ková E. 2010. Conserved epitopes of influenza A virus inducing protective immunity and their prospects for universal vaccine development. Virol J. 7: 351.
9. Sominskaya I, Skrastina D, Dislers A, Vasiljev D, Mihailova M, Ose V, et al. 2010 Construction and immunological evaluation of multivalent hepatitis B virus (HBV) core virus-like particles carrying HBV and HCV epitopes. Clin Vaccine Immunol. 17(6): 1027-33.

10. Sette A, Newman M, Livingston B, McKinney D, Sidney J, Ishioka G. et al. 2002. Optimizing vaccine design for cellular processing, MHC binding and TCR recognition. Tissue Antigens. 59(6): 443-451.

11. Benson DA, Karsch-Mizrachi I, Lipman DJ, Ostell J, Sayers EW. 2009. GenBank. Nucleic Acids Res. 37 (Database issue), D26-D31.

12. Sievers F, Wilm A, Dineen D, Gibson TJ, Karplus K, Li W et al. 2011. Fast, scalable generation of high-quality protein multiple sequence alignments using Clustal Omega. Mol Syst Biol. 7: 539.

13. Waterhouse AM, Procter JB., Martin DM, Clamp M, Barton GJ, 2009. Jalview Version 2-a multiple sequence alignment editor and analysis workbench. Bioinformatics. 25(9): 1189-1191.

14. Shannon CE. 1948. A mathematical theory of communication. Bell Syst Tech J. 27(3): 379-423.

15. Garcia-Boronat M, Diez-Rivero CM, Reinherz EL, Reche PA. 2008. PVS: a web server for protein sequence variability analysis tuned to facilitate conserved epitope discovery. Nucleic Acids Res. 36 (Web Server issue): W35-W41.

16. Doytchinova IA, Flower DR. 2007. VaxiJen: a server for prediction of protective antigens, tumour antigens and subunit vaccines. $B M C$ Bioinform. 8: 4.

17. Kolaskar AS, Tongaonkar PC. 1990. A semi-empirical method for prediction of antigenic determinants on protein antigens. FEBS Lett. 276 (1-2): 172-174.

18. Larsen JE, Lund O, Nielsen M. 2006. Improved method for predicting linear B-cell epitopes. Immunome Res. 2: 2.

19. El-Manzalawy Y, Dobbs D, Honavar V. 2008. Predicting linear B-cell epitopes using string kernels. J. Mol. Recognit. 21(4): 243-255.

20. Bui HH, Sidney J, Li W, Fusseder N, Sette A. 2007. Development of an epitope conservancy analysis tool to facilitate the design of epitope-based diagnostics and vaccines. BMC Bioinform. 8: 361.

21. Vita R, Zarebski L, Greenbaum JA, Emami H, Hoof I, Salimi N et al. 2010. The immune epitope database 2.0. Nucl. Acids Res. 38 (Database issue): D854-D862.

22. Emini EA, Hughes JV, Perlow DS, Boger J.1985. Induction of hepatitis A virus-neutralizing antibody by a virus-specific synthetic peptide. J. Virol. 55(3):836-839.

23. Karplus PA, Schulz GE. 1985. Prediction of chain flexibility in proteins. Naturwissenschaften. 72(4):212-213.

24. Parker JM, Guo D, Hodges RS. 1986. New hydrophilicity scale derived from high-performance liquid chromatography peptide retention data: correlation of predicted surface residues with antigenicity and X-rayderived accessible sites. Biochemistry. 25 (19): 5425-5432.

25. Sharmin R, Islam AB. 2014. A highly conserved WDYPKCDRA epitope in the RNA directed RNA polymerase of human coronaviruses can be used as epitope-based universal vaccine design. BMC Bioinform. 15: 161.

26. Islam R, Sakib MS, Zaman A. 2012. A computational assay to design an epitope-based peptide vaccine against chikungunya virus. Future Virol. 7 (10):1029-1042.

27. Oany AR, Emran AA, Jyoti TP. 2014. Design of an epitope-based peptide vaccine against spike protein of human coronavirus: an in silico approach. Drug Des Devel Ther. 8: 1139-1149.

28. Vlieghe P, Lisowski V, Martinez J, Khrestchatisky M. 2010. Synthetic therapeutic peptides: Science and market. Drug Discov Today. 15(1-2):4056.

29. Gupta S, Kapoor P, Chaudhary K, Gautam A, Kumar R, Raghava GP. 2013. In Silico Approach for Predicting Toxicity of Peptides and Proteins. PLOS ONE. 8(9): e73957 
30. Biasini M, Bienert S, Waterhouse A, Arnold K, Studer G, Schmidt T et al. 2014. SWISS-MODEL: modelling protein tertiary and quaternary structure using evolutionary information. Nucl Acids Res. 42(W1): W252W258.

31. Schwede T, Kopp J, Guex N, Peitsch MC. 2003. SWISS-MODEL: an automated protein homology-modeling server. Nucl. Acids Res. 31(13): $3381-3385$.

32. Laskowski RA, MacArthur MW, Moss DS, Thornton JM. 1993. PROCHECK: a program to check the stereochemical quality of protein structures. J. Appl. Crystallogr. 26(2):283-291.

33. Wiederstein M, Sippl MJ. 2007. ProSA-web: interactive web service for the recognition of errors in three-dimensional structures of proteins. Nucl. Acids Res. 35 (Web Server issue): W407-W410.

34. Xue M, Shi X, Zhang J, Zhao Y, Cui H, Hu S. 2012. Identification of a Conserved B-cell Epitope on Reticuloendotheliosis Virus Envelope Protein by Screening a Phage-displayed Random Peptide Library. PLOS ONE. 7(11): e49842.

35. World Health Organization. Yellow fever-Brazil. Available at: http:// www.who.int/csr/don/09-March-2018-yellow-fever-brazil/en/. Accessed 18 May, 2018.

36. Thomas RE, Lorenzetti DL, Spragins W, Jackson D, Williamson T. 2011. Reporting rates of yellow fever vaccine 17D or 17DD-associated serious adverse events in pharmacovigilance data bases: systematic review. Curr Drug Saf. 6(3):145-54.

37. Lefeuvre A, Marianneau P, Deubel V. 2004. Current Assessment of Yellow Fever and Yellow Fever Vaccine. Curr Infect Dis Rep. 6(2): 96-104.

38. Vasconcelos PF, Luna EJ, Galler R, Silva LJ, Coimbra TL, Barros VL, et al. 2001. Brazilian Yellow Fever Vaccine Evaluation. Serious adverse events associated with yellow fever 17DD vaccine in Brazil: a report of two cases. Lancet. 358 (9276): 91-97.
39. Cetron MS, Marfin AA, Julian KG, Gubler DJ, Sharp DJ, Barwick RS, et al. 2002. Yellow fever vaccine. Recommendations of the Advisory Committee on Immunization Practices (ACIP), 2002. MMWR Recomm Rep. 51(RR-17): 1-11; quiz CE11-14.

40. Maciel M, Cruz F da SP, Cordeiro MT, da Motta MA, Cassemiro KM, S. de M Maia et al. 2015. A DNA Vaccine against Yellow Fever Virus: Development and Evaluation. PLoSNegl Trop Dis. 9(4): e0003693.

41. Arnon R. 2006. A novel approach to vaccine design-epitope-based vaccines. FEBS J. 273:33-34

42. Barros MC, Galasso TG, Chaib AJ, Degallier N, Nagata T, Ribeiro BM. 2011. Yellow fever virus envelope protein expressed in insect cells is capable of syncytium formation in lepidopteran cells and could be used for immunodetection of YFV in human sera. Virol J. 8: 261.

43. Greenbaum JA, Andersen PH, Blythe M, Bui HH, Cachau RE, Crowe J et al., 2007. Towards a consensus on datasets and evaluation metrics for developing B-cell epitope prediction tools. J Mol Recognit. 20(2):75-82.

44. Caoili SEC. 2010. B-cell epitope prediction for peptide-based vaccine design: towards a paradigm of biological outcomes. Proceedings of the First ACM International Conference on Bioinformatics and Computational Biology, Niagara Falls, New York.

45. Novotný J, Handschumacher M, Haber E, Bruccoleri RE, Carlson WB, Fanning DW et al.1986. Antigenic determinants in proteins coincide with surface regions accessible to large probes (antibody domains). Proc Natl Acad Sci U S A. 83(2):226-230.

46. Olesen OF, Lonnoroth A, Mulligan B. 2009. Human vaccine research in the European Union. Vaccine. 27(5):640-645.

47. Guex N, Peitsch MC.1997. SWISS-MODEL and the Swiss-PdbViewer: an environment for comparative protein modeling. Electrophoresis. 18(15): 2714-23. 